\title{
Versatile universal voltage-mode filter employing minimum components
}

\author{
Y. Z. Liao' ${ }^{1}$, H. P. Chen ${ }^{2 a)}$ and W. T. Lee \\ ${ }^{1}$ Graduate Institute of Computer and Communication Engineering, National Taipei \\ University of Technology, Taiwan, ROC \\ ${ }^{2}$ Department of Electronic Engineering and Institute of Electronic Engineering, \\ Ming Chi University of Technology, Taiwan, ROC \\ a)hpchen8888@xuite.net
}

Abstract: A novel versatile single FDCCII-based voltage-mode universal biquadratic filter with three inputs and four outputs is presented. The proposed circuit can act as a multifunction voltage-mode filter with two inputs and four outputs and can perform simultaneous realization of voltage-mode bandreject, highpass, bandpass and lowpass filter signals from the four output terminals, respectively. On the other hand, it also can act as a universal voltage-mode filter with three inputs and a single output and can realize five generic voltage-mode filter signals without requiring any inverting input voltage signals and componentmatching conditions. The proposed circuit employing single FDCCII, two grounded capacitors and two resistors, which are the minimum components necessary for realizing a second-order voltage-mode universal filtering response from the same topology. Both its active and passive sensitivities are low.

Keywords: voltage-mode filter, FDCCII, analog circuit design

Classification: Integrated circuits

\section{References}

[1] B. Wilson, "Recent developments in current conveyor and current-mode circuits," Proceedings of IEE, vol. 137, no. 2, pp. 63-77, 1990.

[2] G. W. Roberts and A. S. Sedra, "All current-mode frequency selective circuits," Electron. Lett., vol. 25, no. 12, pp. 759-761, 1989.

[3] C. M. Chang, "Current mode allpass/notch and bandpass filter using single CCII," Electron. Lett., vol. 27, no. 20, pp. 1812-1813, 1991.

[4] A. Fabre, O. Saaid, F. Wiest, and C. Boucheron, "Low power currentmode second-order bandpass IF filter," IEEE Trans. Circuits Syst. II, Analog Digit. Signal Process., vol. 44, no. 6, pp. 436-446, 1997.

[5] M. A. Ibrahim, S. Minaei, and H. Kuntman, "A 22.5 MHz current-mode KHN-biquad using differential voltage current conveyor and grounded passive elements," Int. J. Electron. Commun. (AË̈), vol. 59, no. 5, pp. 311-318, 2005. 
[6] A. A. El-Adwy, A. M. Soliman, and H. O. Elwan, "A novel fully differential current conveyor and applications for analog VLSI," IEEE Trans. Circuits Syst. II, Analog Digit. Signal Process., vol. 47, no. 4, pp. 306313, 2000.

[7] C. M. Chang and M. J. Lee, "Voltage-mode multifunction filter with single input and three outputs using two compound current conveyors," IEEE Trans. Circuits Syst. I, Fundam. Theory Appl., vol. 46, no. 11, pp. 1364-1365, 1999.

[8] J. W. Horng, W. Y. Chui, and H. Y. Wei, "Voltage-mode highpass, bandpass and lowpass filters using two DDCCs," Int. J. Electron., vol. 91, no. 8, pp. 461-464, 2004.

[9] H. P. Chen and P. L. Chu, "Versatile voltage-mode multifunction biquadratic filter employing DDCCs," IEICE Electron. Express, vol. 5, no. 18 , pp. 769-775, 2008.

[10] C. M. Chang, B. M. Al-Hashimi, C. L. Wang, and C. W. Hung, "Single fully differential current conveyor biquad filters," IEE Proc. Circuit, Device Syst., vol. 150, no. 5, pp. 394-398, 2003.

[11] M. Bhushan and R. W. Newcomb, "Grounding of capacitors in the integrated circuits," Electron. Lett., vol. 3, no. 4, pp. 148-149, 1967.

[12] R. Senani, "New universal current mode biquad employing all grounded passive components but only two DOCCs," J. Active and Passive Electron. Device, vol. 1, no. 3-4, pp. 281-288, 2006.

[13] C. M. Chang and H. P. Chen, "Universal capacitor-grounded voltagemode filter with three inputs and a single output," Int. J. Electron., vol. 90, no. 6, pp. 401-406, 2003.

[14] J. W. Horng, C. L. Hou, C. M. Chang, H. P. Chou, C. T. Lin, and Y. H. Wen, "Quadrature oscillators with grounded capacitors and resistors using FDCCIIs," ETRI Journal, vol. 28, no. 4, pp. 486-494, 2006.

\section{Introduction}

High performance active circuits have received much attention. Since currentmode circuits offer the main advantages like greater linearity, lower power consumption and wider bandwidth over their voltage-mode counterparts $[1,2$, 3]. Also, the design of filter circuits employing current-mode active elements may be used in phase-locked look frequency modulation stereo demodulators, touch-tone telephone tone decoder and cross-over networks in a threeway high fidelity loudspeaker $[4,5]$. In 2000, a new active element called the fully differential current conveyor (FDCCII) has been proposed [6] to improve the dynamic range in mixed-mode applications where fully differential signal processing is required. The application of FDCCII in filter design was also demonstrated in [6], where three filter biquads were described. Each biquad employs three FDCCIIs, two grounded capacitors and three resistors, which have been implemented using MOS transistors. From the wealth of knowledge on RC active filters, it is known that it is possible to design filter biquadratic using a single active element, two resistors and two capacitors. In 1999, Chang and Lee proposed a voltage-mode highpass (HP), bandpass (BP) and lowpass (LP) biquadratic filter responses with single input and three outputs using two compound current conveyors, two grounded capacitors and three 


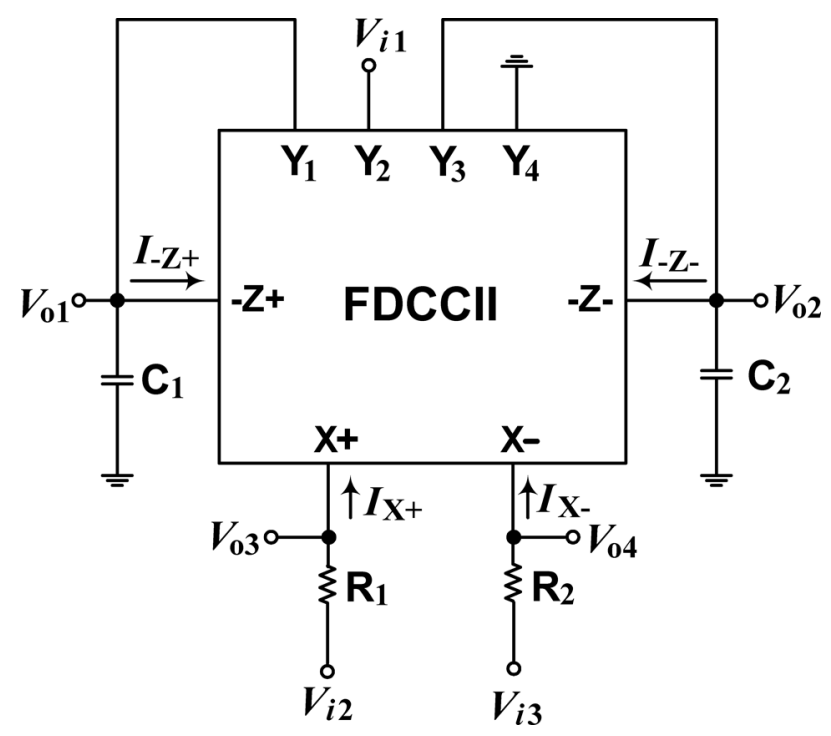

Fig. 1. The proposed versatile universal voltage-mode biquad.

resistors [7]. Then, Horng et al. proposed another voltage-mode HP, BP and LP biquadratic filter responses with single input and three outputs using two differential difference current conveyors (DDCCs), two grounded capacitors and two grounded resistors [8]. However, these two proposed configurations required at least two (not one) active components. Recently, the authors propose a new versatile voltage multifunction biquadratic filter employing two DDCCs, two grounded capacitors and two resistors [9]. However, it still employs two active components. Although, the multifunction filter with single input and four outputs was proposed by Chang et al. [10]. Only three HP, BP and LP biquadratic filter responses can be simultaneously obtained. This paper describes a new minimum components filter biquad based on the recently introduced FDCCII. The proposed circuit can act as a multifunction voltage-mode filter with two inputs and four outputs and can perform simultaneous realization of voltage-mode bandreject (BR), highpass, bandpass and lowpass filter responses from the four output terminals, respectively. Therefore, it is one more BR response than the recent one proposed by Chang et al. [10]. On the other hand, it also can act as a universal voltage-mode filter with three inputs and a single output and can realize five generic voltagemode filter responses from the same configuration. It can not be realized as reported by Chang et al. [10].

\section{Circuit description}

The FDCCII is an eight-terminal analog building block and its terminal relations are given by [10] 


$$
\left[\begin{array}{c}
V_{X+} \\
V_{X-} \\
I_{-Z+} \\
I_{-Z-}
\end{array}\right]=\left[\begin{array}{cccccc}
0 & 0 & 1 & -1 & 1 & 0 \\
0 & 0 & -1 & 1 & 0 & 1 \\
-1 & 0 & 0 & 0 & 0 & 0 \\
0 & -1 & 0 & 0 & 0 & 0
\end{array}\right]\left[\begin{array}{l}
I_{X+} \\
I_{X-} \\
V_{Y 1} \\
V_{Y 2} \\
V_{Y 3} \\
V_{Y 4}
\end{array}\right]
$$

The proposed versatile universal-mode circuit comprises single FDCCII, two grounded capacitors and two resistors, as shown in Fig. 1. The use of grounded capacitors makes the circuit suitable for integration because grounded capacitors circuit can compensate for the stay capacitances at their nodes $[11,12,13]$. Derived by each nodal equation of the proposed circuit, the input-output relationship matrix form of Fig. 1 can be express as

$$
\left[\begin{array}{cccc}
s C_{1} & 0 & G_{1} & 0 \\
0 & s C_{2} & 0 & G_{2} \\
-1 & -1 & 1 & 0 \\
1 & 0 & 0 & 1
\end{array}\right]\left[\begin{array}{c}
V_{o 1} \\
V_{o 2} \\
V_{o 3} \\
V_{o 4}
\end{array}\right]=\left[\begin{array}{c}
G_{1} V_{i 2} \\
G_{2} V_{i 3} \\
-V_{i 1} \\
V_{i 1}
\end{array}\right]
$$

$G_{1}=\frac{1}{R_{1}}$ and $G_{2}=\frac{1}{R_{2}}$.

To derive Eq. (2), all four $Y_{1}, Y_{2}, Y_{3}$ and $Y_{4}$ terminals of FDCCII are high-impedance terminals, since there are connected to gates of MOS devices in actual implementation, whereas the two ports $X+$ and $X-$ are low impedance terminals [6]. Similarly, the two ports $-Z+$ and $-Z-$ also exhibit high impedance since there are connected to the output stages of current mirrors [6]. From Eq. (2), the following four output voltages can be derived as

$$
\begin{gathered}
V_{o 1}=\frac{1}{\triangle}\left[\left(s C_{2} G_{1}+G_{1} G_{2}\right) V_{i 1}+\left(s C_{2} G_{1}\right) V_{i 2}-\left(G_{1} G_{2}\right) V_{i 3}\right] \\
V_{o 2}=\frac{1}{\triangle}\left[-\left(s C_{1} G_{2}\right) V_{i 1}+\left(G_{1} G_{2}\right) V_{i 2}+\left(s C_{1} G_{2}+G_{1} G_{2}\right) V_{i 3}\right] \\
V_{o 3}=\frac{1}{\triangle}\left[-\left(s^{2} C_{1} C_{2}+s C_{1} G_{2}\right) V_{i 1}+\left(s C_{2} G_{1}+G_{1} G_{2}\right) V_{i 2}+\left(s C_{1} G_{2}\right) V_{i 3}\right] \\
V_{o 4}=\frac{1}{\triangle}\left[\left(s^{2} C_{1} C_{2}\right) V_{i 1}-\left(s C_{2} G_{1}\right) V_{i 2}+\left(G_{1} G_{2}\right) V_{i 3}\right] \\
\triangle=s^{2} C_{1} C_{2}+s C_{2} G_{1}+G_{1} G_{2} .
\end{gathered}
$$

Depending on the status of the biquad input three voltages: $V_{i 1}, V_{i 2}$ and $V_{i 3}$, numerous filter functions are obtained. There are two cases shown as below.

Case I. If $V_{i 1}=V_{i 3}=V_{i n}$ (the input voltage signal) and $V_{i 2}=0$, then

$$
\begin{aligned}
& \frac{V_{o 1}}{V_{i n}}=\frac{s C_{2} G_{1}}{s^{2} C_{1} C_{2}+s C_{2} G_{1}+G_{1} G_{2}} . \\
& \frac{V_{o 2}}{V_{i n}}=\frac{G_{1} G_{2}}{s^{2} C_{1} C_{2}+s C_{2} G_{1}+G_{1} G_{2}} . \\
& \frac{V_{o 3}}{V_{i n}}=\frac{-s^{2} C_{1} C_{2}}{s^{2} C_{1} C_{2}+s C_{2} G_{1}+G_{1} G_{2}} .
\end{aligned}
$$




$$
\frac{V_{o 4}}{V_{\text {in }}}=\frac{s^{2} C_{1} C_{2}+G_{1} G_{2}}{s^{2} C_{1} C_{2}+s C_{2} G_{1}+G_{1} G_{2}} .
$$

Thus, the non-inverting bandpass, non-inverting lowpass, inverting highpass, and non-inverting bandreject signals are obtained at the node voltages, $V_{o 1}, V_{o 2}, V_{o 3}$ and $V_{o 4}$, respectively.

Case II. The specializations of the numerator in equation (6) yield the five types of biqudratic filters with the following specialization.

(i) lowpass: $V_{i 1}=V_{i 2}=0$ and $V_{i 3}=V_{i n}$;

(ii) bandpass: $V_{i 1}=V_{i 3}=0$ and $V_{i 2}=V_{i n}$;

(iii) highpass: $V_{i 2}=V_{i 3}=0$ and $V_{i 1}=V_{i n}$;

(iv) bandreject: $V_{i 2}=0$ and $V_{i 1}=V_{i 3}=V_{i n}$;

(v) allpass: $V_{i 1}=V_{i 2}=V_{i 3}=V_{i n}$.

Note that there is also no need of any component-matching conditions and inverting-type voltage input signals to realize all of the filter responses.

Obviously, from cases I and II, the proposed circuit can act as a multifunction voltage-mode filter with two inputs and four outputs and can perform simultaneous realization of voltage-mode bandreject, highpass, bandpass and lowpass filter signals from the four output terminals, respectively. Moreover, it also can act as a universal voltage-mode filter with three inputs and a single output and can realize five generic voltage-mode filter signals without requiring any inverting input voltage signals and component-matching conditions.

\section{Effect of Non-idealities}

Taking into account the non-idealities of an FDCCII, equation (1) becomes

$$
\left[\begin{array}{c}
V_{X+} \\
V_{X-} \\
I_{-Z+} \\
I_{-Z-}
\end{array}\right]=\left[\begin{array}{cccccc}
0 & 0 & \alpha_{a} & -\alpha_{b} & \alpha_{c} & 0 \\
0 & 0 & -\alpha_{d} & \alpha_{e} & 0 & \alpha_{f} \\
-\beta_{a} & 0 & 0 & 0 & 0 & 0 \\
0 & -\beta_{b} & 0 & 0 & 0 & 0
\end{array}\right]\left[\begin{array}{c}
I_{X+} \\
I_{X-} \\
V_{Y 1} \\
V_{Y 2} \\
V_{Y 3} \\
V_{Y 4}
\end{array}\right]
$$

$\alpha_{v}=1-e_{v}$ and $e_{v}\left(\left|e_{v}\right| \ll 1\right)$ denote the voltage tracking error, and $\beta_{i}=1-e_{i}$ and $e_{i}\left(\left|e_{i}\right| \ll 1\right)$ denote the differential current tracking error. The denominator of the transfer functions of Fig. 1 becomes

$$
D(s)=s^{2} C_{1} C_{2}+\alpha_{a} \beta_{a} s C_{2} G_{1}+\alpha_{c} \alpha_{d} \beta_{a} \beta_{b} G_{1} G_{2} .
$$

The non-ideal $\omega_{o}$ and $Q$ are given by

$$
\begin{aligned}
& \omega_{o}=\sqrt{\frac{\alpha_{c} \alpha_{d} \beta_{a} \beta_{b} G_{1} G_{2}}{C_{1} C_{2}} .} \\
& Q=\frac{1}{\alpha_{a}} \sqrt{\frac{\alpha_{c} \alpha_{d} \beta_{b} C_{1} G_{2}}{\beta_{a} C_{2} G_{1}}} .
\end{aligned}
$$




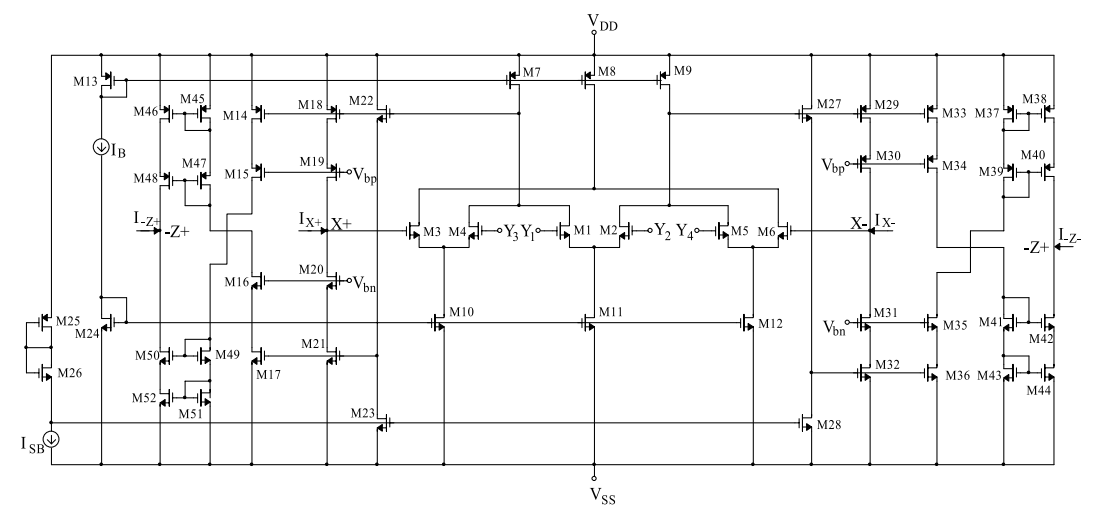

Fig. 2. The CMOS implementation of a FDCCII.

A sensitivity study forms an important index of the performance of any active network. The formal definition of sensitivity is

$$
S_{x}^{F}=\frac{x}{F} \frac{\partial F}{\partial x},
$$

where $F$ represents one of $\omega_{o}, Q$ and $x$ represents any of the passive elements $\left(G_{1}-G_{2}, C_{1}-C_{2}\right)$ or the active parameters $\left(\alpha_{i}, \beta_{i}\right)$. Using the above definition the active and passive sensitivities of the proposed circuit shown in Fig. 1 are given as

$$
\begin{gathered}
S_{\alpha_{c}}^{\omega_{o}}=S_{\alpha_{d}}^{\omega_{o}}=S_{\beta_{a}}^{\omega_{o}}=S_{\beta_{b}}^{\omega_{o}}=S_{G_{1}}^{\omega_{o}}=S_{G_{2}}^{\omega_{o}}=-S_{C_{1}}^{\omega_{o}}=-S_{C_{2}}^{\omega_{o}}=\frac{1}{2} ; \\
S_{\alpha_{c}}^{Q}=S_{\alpha_{d}}^{Q}=S_{\beta_{b}}^{Q}=S_{C_{1}}^{Q}=S_{G_{2}}^{Q}=-S_{\beta_{a}}^{Q}=-S_{C_{2}}^{Q}=-S_{G_{1}}^{Q}=\frac{1}{2} ; \\
S_{\alpha_{a}}^{Q}=-1 .
\end{gathered}
$$

Hence, the filter parameter sensitivities are low and not larger than unity in absolute value.

\section{Simulation results}

Finally, to verify the theoretical prediction of the proposed biquad filter, we use the HSPICE for the simulation part with a $0.35 \mu \mathrm{m}$ process and Matlab for the theoretical part to compare the results. The CMOS implementation of the FDCCII is shown in Fig. $2[6,14]$, which was slightly modified by using current mirrors to take the current outputs $-\mathrm{Z}+$ and $-\mathrm{Z}$ - terminals. The NMOS and PMOS transistor aspect ratios are given by $(W / L=5 \mu \mathrm{m} / 1 \mu \mathrm{m})$ and $(W / L=10 \mu \mathrm{m} / 1 \mu \mathrm{m})$, respectively. The supply voltages are $V_{D D}=$ $-V_{S S}=1.65 \mathrm{~V}$, the biasing voltages are $V_{b n}=-V_{b p}=0 \mathrm{~V}$, and the biasing currents are $I_{B}=I_{S B}=50 \mu \mathrm{A}$. The proposed circuit was designed for $f_{o}=1 \mathrm{MHz}$ and $Q=1$ by choosing $R_{1}=10 \mathrm{k} \Omega, R_{2}=10 \mathrm{k} \Omega, C_{1}=15.9 \mathrm{pF}$ and $C_{2}=15.9$ pF. Fig. 3 (a) shows the simulated results of LP, BP, HP and $\mathrm{BR}$ amplitude-frequency responses with $V_{i 1}=V_{i 3}=V_{i n}$ and $V_{i 2}=0$. As can be seen, there is a close agreement between theory and simulation. To test the input dynamic range of the proposed circuit, the simulation has been repeated for a sinusoidal input signal at $f_{o}=1 \mathrm{MHz}$. Fig. 3 (b) shows that the input dynamic range of the $\mathrm{BP}$ response at $V_{o 4}$ output terminal with $V_{i 2}=V_{i n}$ and $V_{i 1}=V_{i 3}=0$, which extends up to amplitude of $1.2 \mathrm{~V}$ (peak to peak) without signification distortion. 


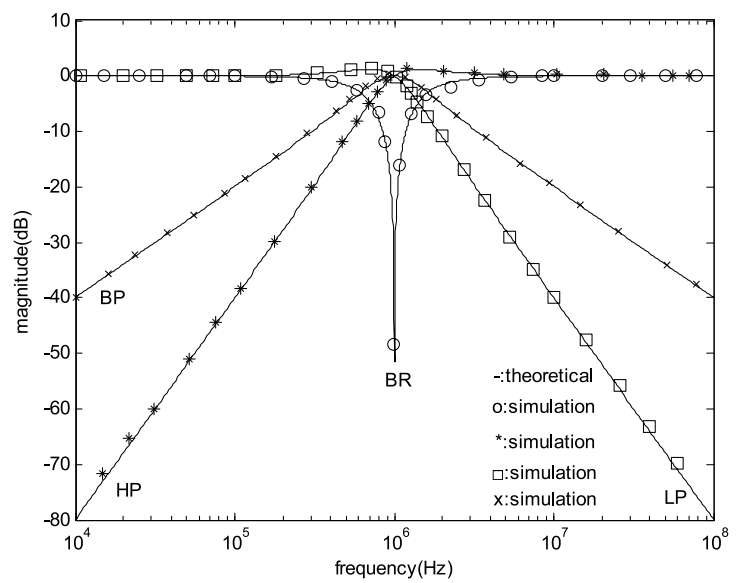

(a)

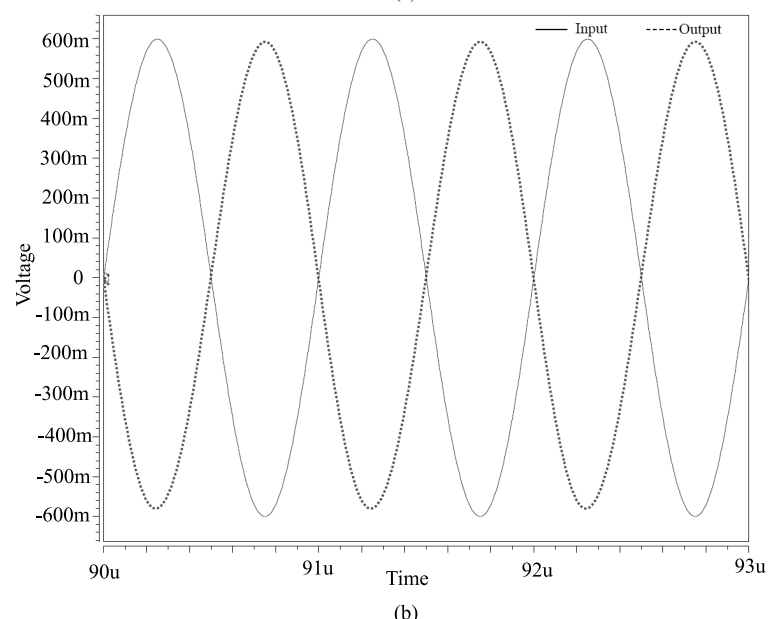

Fig. 3. (a) The simulated results of LP, BP, HP and BR amplitude-frequency responses. (b) The input and output waveforms of the BP response for a $1 \mathrm{MHz}$ sinusoidal input voltage of $1.2 \mathrm{~V}$ (peak to peak).

\section{Conclusion}

In 2003, Chang et al. presented a voltage-mode multifunction filter with single input and three outputs employing single FDCCII, two grounded capacitors and two grounded resistors [10]. It can simultaneously obtain HP, $\mathrm{BP}$ and LP filter responses. In this paper, the authors also proposed a single FDCCII-based voltage-mode universal biquadratic filter with three inputs and four outputs. The proposed circuit can be used as either a two-input four-output multifunction or a three-input single-output universal filter with the same topology. Because it is more versatile than the multifunction one proposed by Chang et al. [10]. Moreover, in addition to HP, BP and LP responses, the proposed circuit can also realize BR and AP filter responses.

\section{Acknowledgments}

This work was supported by National Science Council, Republic of China, under Grant NSC-98-2221-E-131-027. 